\title{
Excentricité et forme des sections transversales de bois. Définitions, méthodologie, exemples sur l'épicéa commun (Picea abies Karst.)
}

\author{
Laurent Saint-André \\ Équipe de recherches sur la qualité des bois, Inra, 54280 Champenoux, France
}

(Reçu le 10 octobre 1998 ; accepté le 12 février 1998)

\begin{abstract}
Asymmetry of wood cross-sections. Definitions, methodology, and case study on Norway-spruce. This study makes a revue of different definitions used to characterise the asymmetry of transverse sicm sections. When the shape is described by intersection with $2 n$ radii (one every $180 / n$ degrees), the centre of mass of the points, the surface, and the shape can be defined. These points depend on the number and the orientation of the radii. Their use for calculating eccentricity (vector between the pith and the centre of the cross-section) or ellipticity (ratio between the largest diameter to its perpendicular) is tested in various cases. An example is given for simple geometric shapes (circle, ellipse) and for 168 Norway spruce cross-sections. (@) Inra/Elsevier, Paris.)
\end{abstract}

\section{form / eccentricity / centre of mass / geometry / Picea abies Karst.}

Résumé - Cette note fait le point sur plusieurs définitions importantes lorsqu'il s'agit de caractériser l'asymétrie de la croissance du bois en section transversale. Lorsque le contour des rondelles est discrétisé par intersection avec un certain nombre de rayons pairs $(2 n)$ équirépartis en direction sur $[0,2 \pi]$ et originaires de la moelle, il est possible de définir le centre de gravité des points, de la surface et du contour. Ces points sont généralement distincts et sensibles au nombre de rayons utilisés ainsi qu'à leurs orientations. Pour calculer l'excentricité (position de la moelle par rapport au centre de la rondelle), ou le méplat (rapport entre le plus grand diamètre et son perpendiculaire, passants tous deux par le centre de la rondelle) l'utilisation de l'un ou l'autre de ces points donne des résultats très différents. Les applications sont réalisćes pour des figures géométriques simples (cercle, ellipse) et pour 168 rondelles réelles de bois (épicéa commun). (@ Inra/Elsevier, Paris.)

forme / excentricité / centre de gravité / barycentre / Picea abies Karst.

\footnotetext{
* Correspondance el tirés à part.

e-mail : s.andre@nancy.inra.fr
} 


\section{INTRODUCTION}

Si plusieurs études concernent la forme du tronc dans le sens longitudinal tant sur le défilement $[9,15]$ que sur l'inclinaison des tiges $[13,18]$, relativement peu de travaux sont consacrés à la forme ou à l'excentricité de la moelle des sections transversales de la tige. D'une manière générale, les vents dominants sont souvent décrits comme responsables de ces défauts. Williamson [20] utilise cette hypothèse pour expliquer la non circularité des tiges de Douglas après n'avoir constaté que peu de relations avec l'inclinaison de l'arbre ou le déséquilibre du houppier. Dans le même sens, Laar [11] ne constate pas de relations entre la forme des sections (sur différentes essences feuillues et résineuses) et la pente ou la densité des peuplements, par contre le plus grand axe des rondelles coïncide avec la direction des vents dominants. Bouillet et Houllier [6] montrent enfin que les éclaircies n'influent pas sur l'excentricité des tiges de Pinus kesiya.

En terme de qualité des bois, les défauts de forme ou d'excentricité se traduisent systématiquement par une hétérogénéité du bois, notamment par des largeurs de cernes plus ou moins grandes et souvent par la présence de bois de réaction dont les qualités technologiques sont amoindries [7, 13]. Ce bois induit d'une part une déformation des pièces plus importante au séchage (retraits longitudinaux plus forts et retraits tangentiels plus faibles), et d'autre part des cassures au déroulage du fait des méats intercellulaires [10]. La forme des sections transversales a souvent fait l'objet de simplifications dans les logiciels d'optimisation de débit et de représentation des grumes [3,12], mais peu de mesures ont été réalisées pour étayer ces suppositions (rondelles circulaires ou elliptiques avec symétrie axiale).

Enfin, ces problèmes d'excentricité et de forme intéressent aussi le dendrométricien et le dendrochronologiste, parce qu'elles peuvent induire des biais et, ou des erreurs, sur les estimations de surface terrière ou de largeurs des cernes $[2,14,19]$.

D'une manière générale, les études sur l'asymétrie de croissance des tiges présentent une confusion dans la définition des termes et surtout dans la mesure de cette caractéristique. Par exemple, pour la même définition du méplat (ellipticity), c'est-àdire le rapport entre le plus grand diamètre de la rondelle et son perpendiculaire, Boissieras [4] impose le passage du plus grand diamètre par la moelle, tandis que Williamson (Op. Cit.) ne s'en préoccupe pas. Il est évident, que dans le premier cas le méplat sera lié à l'excentricité des rondelles, tandis que dans le second, on a effectivement une mesure de la forme indépendante de la position de la moelle.

L'objet de cette note est de proposer une définition plus générale des termes utilisés pour décrire l'asymétrie des sections puis de donner une méthode de calcul de l'excentricité et du méplat à partir des analyses de tiges classiques.

\section{DÉFINITIONS}

\subsection{Barycentre, centre de gravité}

Que ce soit en mathématique ou en physique, le barycentre (B) d'un système de $n$ points $\mathrm{Ai}$, chacun affecté de la masse $\mathrm{Pi}$, est défini par la relation vectorielle suivante :

$$
\sum_{i=1}^{n} P_{i} \overrightarrow{B A}_{i}=\overrightarrow{0}
$$

Lorsque les points ont tous une masse identique, B est le centre de gravité de ce système de points.

Dans le cas d'une ligne ou d'une surface, systèmes continus par définition, le centre de gravité est calculé en décomposant puis en pondérant par la longueur ou la surface de chaque sous-ensemble. La figure I montre que pour une même forme d'objet, ici un 


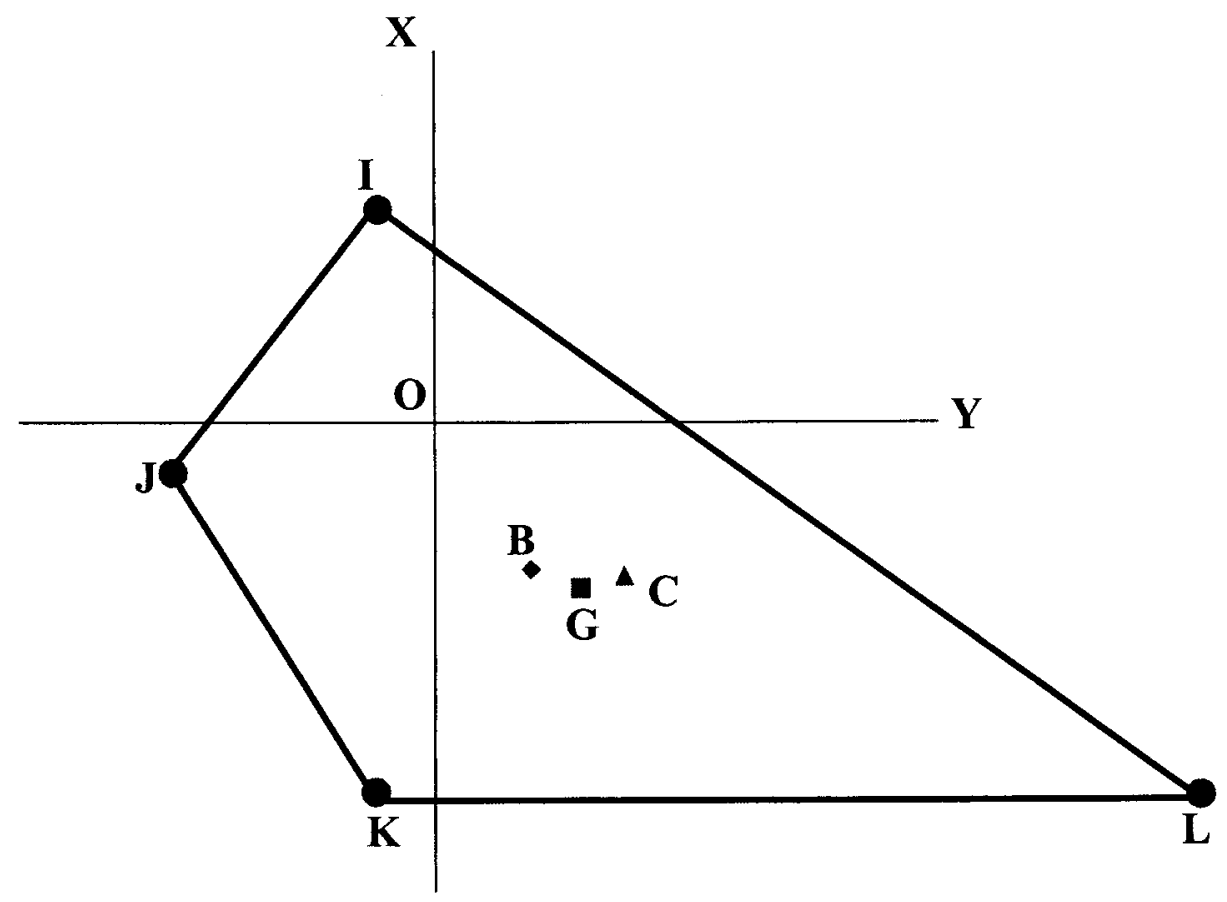

Figure 1. Un quadrilatère IJKL et trois centres de gravité. B est celui des points, C celui du contour, G celui de la surface.

quadrilatère, le centre de gravité des points (B), du contour (C) et de la surface (G) sont distincts.

\subsection{Excentricité, méplat}

Après une synthèse bibliographique, Bouillet [5] aboutit aux concepts suivants : l'excentricité désigne l'écart entre la position de la moelle et le « barycentre » (en l'occurrence le centre de gravité des points utilisés pour décrire le contour de la rondelle), tandis que le méplat décrit l'aplatissement de cette rondelle.

Ces concepts présentent l'avantage de bien séparer la forme de la section (dont un critère est le méplat), de l'excentricité qui est la position de la moelle dans la rondelle. En revanche, pour décrire correctement l'asymétrie des sections transversales de bois et son évolution au cours du temps, le choix d'utiliser l'un ou l'autre des trois centres de gravité décrits précédemment n'est pas anodin. Dans le cadre de cette note, le point de référence sera le centre de gravité de la surface de la rondelle. La motivation essentielle est que la surface est beaucoup utilisée pour décrire l'accroissement biologique des tiges.

En généralisant la définition proposée cidessus, l'excentricité désigne donc la position de la moelle $(\mathrm{M})$ par rapport au centre de gravité de la surface de la rondelle (G) et s'exprime par le vecteur $\overrightarrow{M G}$. Le méplat est un indicateur de forme qui s'exprime par le rapport entre le plus grand diamètre de la rondelle (D1) et son perpendiculaire (D2), qui passent tous deux par G. (figure 2). 


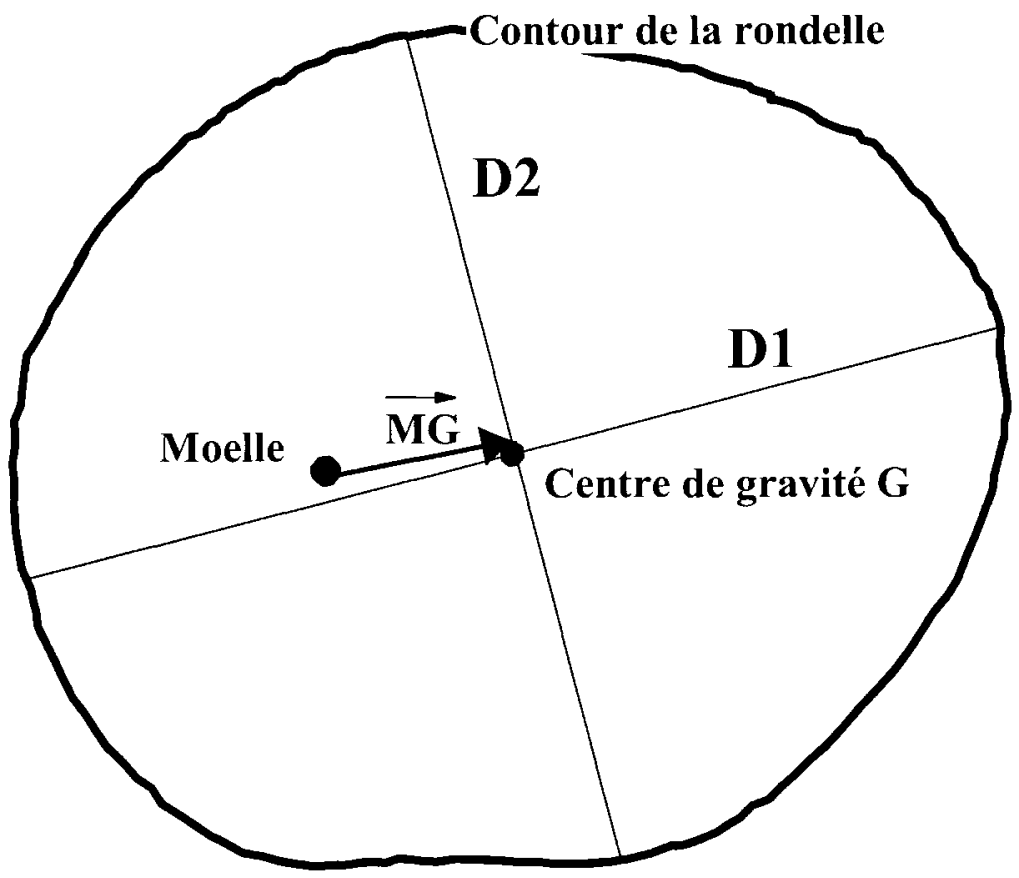

Figures 2. Définition du méplat (D1/D2) et de l'excentricité $\overrightarrow{M G}$, pour une rondelle quelconque.

\subsection{Estimateurs du centre de gravité de la surface et du contour d'une rondelle}

Dans le cas des analyses de tiges, les mesures sont généralement effectuées sur un certain nombre pair de rayons $(2 n)$ équirépartis en direction sur $[0,2 \pi]$ et originaires de la moelle. L'intersection de ces rayons avec le contour de la rondelle donne un système de $2 n$ points Ai. La relation habituellement utilisée pour définir « le centre de gravité » de la rondelle est la suivante $[6$, 181 :

$$
\sum_{i=1}^{2 n} \overrightarrow{B A}_{i}=\overrightarrow{0}
$$

Il s'agit en fait du centre de gravité des points $\mathrm{Ai}$ et non du centre de gravité de la surface $G$ dont un estimateur (G') est défini par la formule suivante :

$$
\sum_{i=1}^{2 n} S_{M i_{i} i+1} \vec{G}^{\prime} T_{M A_{i} i+1}=\overrightarrow{0}
$$

Avec $\mathrm{S}_{\mathrm{MA}_{i} \mathrm{~A}_{i+1}}$ et $\mathrm{T}_{\mathrm{MA}_{\mathrm{i} \mathrm{A}_{i+1}} \text { la surface et le }}$ centre de gravité des triangles $\mathrm{MA}_{\mathrm{i}} \mathrm{A}_{\mathrm{i}}+\mathrm{l}$. G'est le centre de gravité de la surface du polygone ayant pour sommets les points $\mathrm{Ai}$.

De même, du fait de la discrétisation du contour en $2 n$ points, un estimateur ( $\left.C^{\prime}\right) \mathrm{du}$ centre de gravité du contour $(\mathrm{C})$ de la rondelle est donné par la relation suivante:

$$
\sum_{i=1}^{2 n} L_{A_{i^{\prime} i+1}} \vec{G}_{I^{\prime} I_{i^{\prime} i+1}}=\overrightarrow{0}
$$


Avec $\mathrm{L}_{\mathrm{A}_{i} \mathrm{~A}_{i+1}}$ et $\mathrm{I}_{\mathrm{A}_{i} \mathrm{~A}_{i+1}}$, la longueur et le milieu des segments $A_{i} A_{i+1}$. C'est le centre de gravité du contour du polygone ayant pour sommets les points $\mathrm{Ai}$.

Ces trois points (B, G', C') qui sont par définition dépendants du système de rayons choisi, seront étudiés dans le cas du cercle et de l'ellipse (figures géométriques simples) et des rondelles de bois (épicéa commun).

\section{3. ÉTUDE DES ESTIMATEURS DANS LE CAS DU CERCLE ET DE L'ELLIPSE}

La figure 3 représente un cercle de centre $\mathrm{O}$ dans lequel on choisit un point $\mathrm{M}$ quelconque. Ce point symbolisera la moelle. Les triangles blancs représentent les points d'intersection entre le contour et 36 rayons équirépartis en direction et originaires de la moelle. Pour chacun des neuf quadruplets de rayons orthogonaux pris parmi les 36 , les centres de gravité de la surface $\left(G^{\prime}(4)\right)$ et du contour $\left(C^{\prime}(4)\right)$ sont représentés respectivement par des triangles et des points.

On démontre que $\mathrm{B}$ est le milieu de $\mathrm{OM}$ quels que soient le nombre et l'orientation des rayons utilisés. En d'autres termes, la multiplication des rayons ne rapprochera jamais B du centre du cercle. Par contre, les centres de gravité de la surface G'et du contour C'tendent à se rapprocher de $\mathrm{O}$ lorsque le nombre de rayons augmente (G'(36) et $C^{\prime}(36)$ sont pratiquement confondus avec le centre du cercle même si $M$ en est assez éloignée). La convergence est plus rapide pour C'mais cet estimateur est sensible à l'orientation des rayons : $C^{\prime}(4)$ forme un petit nuage de points, ce qui n'est pas le cas pour G'(4). On démontre que quel que soit l'orientation des quatre rayons perpendiculaires, $G^{\prime}(4)$ est fixe et vérifie la relation

$$
\overrightarrow{\mathrm{OG}^{\prime}}(4)=\frac{1}{3} \overrightarrow{\mathrm{OM}}
$$

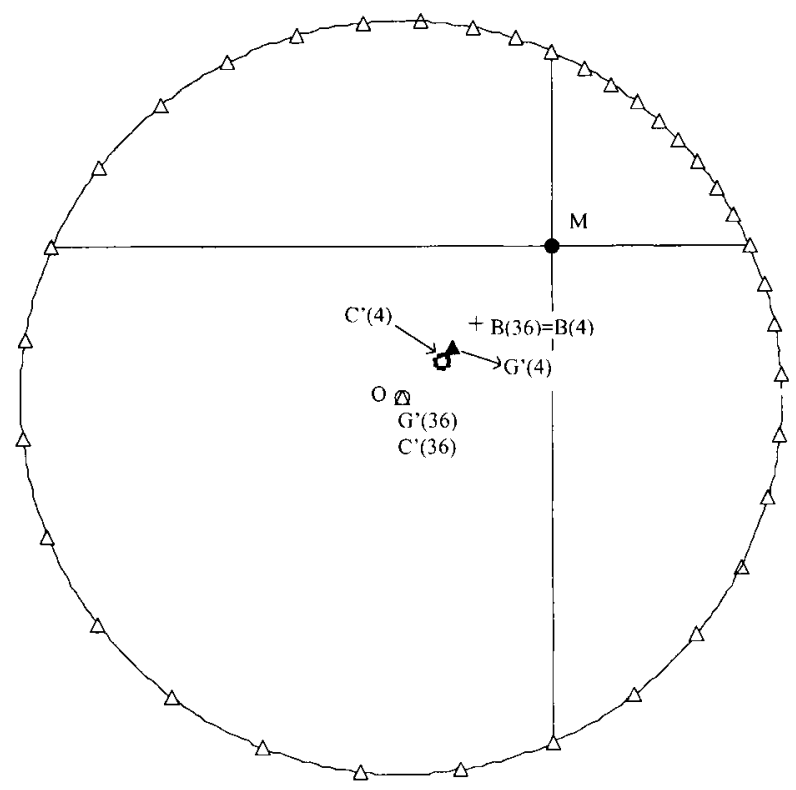

Figure 3. Position du centre de gravité des points (B), de la surface ( $G^{\prime}$ ) et du contour ( $\left.C^{\prime}\right)$ dans le cas du cercle. Le nombre de rayons utilisé pour calculer ces points figure entre parenthèses. 
La figure 4 montre que dans le cas d'une ellipse, les résultats sont similaires : Le centre de gravité des points (B) dépend de la position de M. Il est distinct du centre de l'ellipse et la multiplication des rayons ne l'en rapprochera pas. Par contre, G' (36) et C'(36) sont une nouvelle fois pratiquement confondus avec $\mathrm{O}$.

Le méplat de l'ellipse, et le faible nombre de rayons utilisés font que les trois estimateurs (B (4), C'(4), et G'(4)) sont sensibles à l'orientation des rayons. Cette sensibilité est beaucoup plus marquée pour le centre de gravité du contour (C').

\section{4. ÉTUDE DES ESTIMATEURS DANS LE CAS DES RONDELLES DE BOIS. CONSÉQUENCES SUR L'EXCENTRICITÉ ET LE MÉPLA'T}

\subsection{Matériel}

Pour cette étude, 168 rondelles d'épicéa commun ont été échantillonnées sur 84 grumes en scierie (une rondelle en haut et une en bas). Ces rondelles présentent un diamètre compris entre 10 et $60 \mathrm{~cm}$, pour un nombre de cernes compris entre 11 et 110 ans.

Chaque contour est décrit par ses intersections avec 36 rayons équirépartis en direction et partant de la moelle de la rondelle. Pour chaque rondelle, sont calculés le centre de gravité des 36 points (B), puis les estimateurs ( $G$ 'et $C^{\prime}$ ) des centres de gravité de la surface et du contour à partir des mêmes 36 points. Les distances entre la moelle et ces trois " barycentres » sont représentées sur les figures 5 .

Dans le cas de la figure $5 a$, la droite correspond à $\mathrm{y}=\mathrm{x} / 2$ (relation démontrée sur le cercle). Les points s'écartent peu de la droite car les rondelles sont moins méplates que l'ellipse précédente (représentée par le triangle sur la figure $5 a$ ). En ce qui concerne la position du centre de gravité du contour (carrés blancs), la figure $5 b$ montre qu'il se situe à la même distance de la moelle que le centre de gravité de la surface. La distance entre ces deux points est très faible : en moyenne $0,4 \mathrm{~mm}$, pour un écart type de $0,8 \mathrm{~mm}$ alors que la distance moyenne

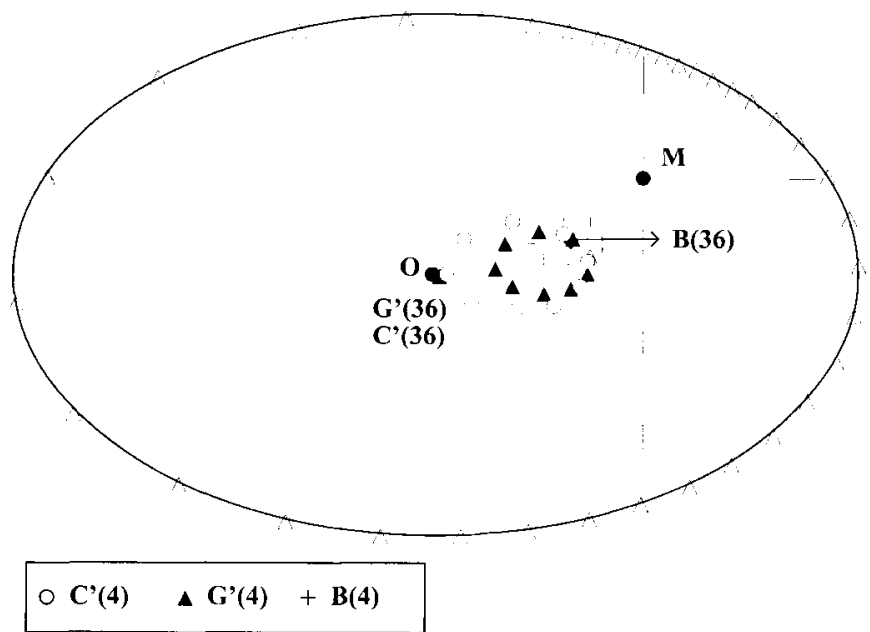

Figure 4. Position du centre de gravité des points (B), de la surface (G') el du contour ( $\left.C^{\prime}\right)$ dans le cas de l'ellipse. Le nombre de rayons utilisé pour calculer ces points figure entre parenthèses. 

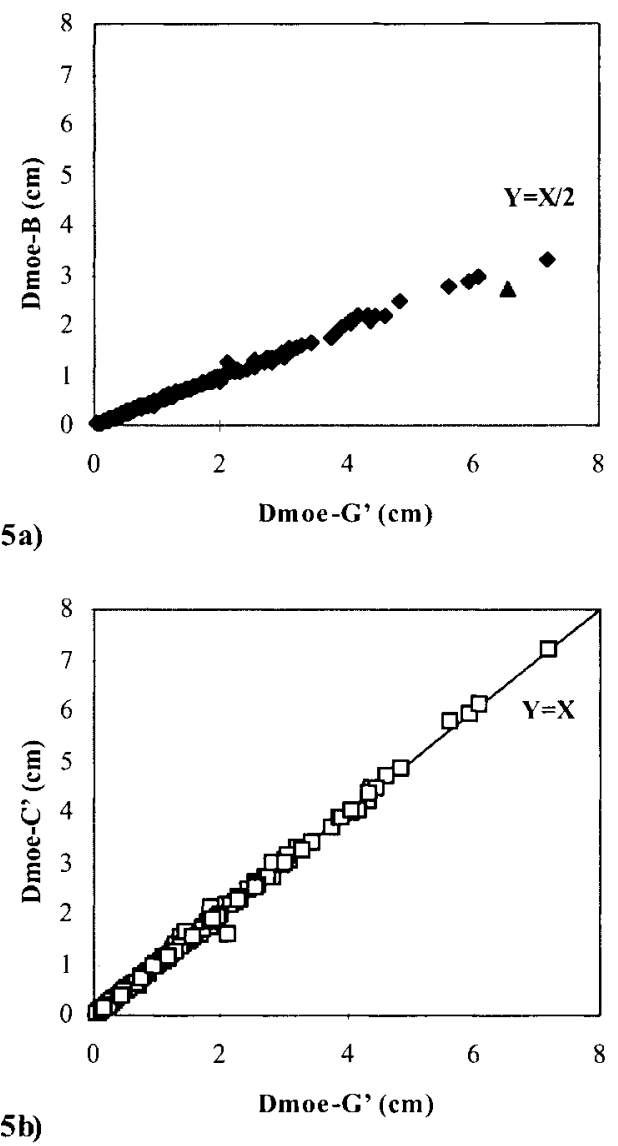

Figures 5. Distance de la moelle aux centres de gravité de la surface (Dmoe-G'), du contour (Dmoe$\mathrm{C}^{\prime}$ ) et des points (Dmoe-B) sur 168 rondelles; les centres de gravité sont estimés à l'aide de 36 rayons équirépartis en angle et partants de la moelle.

a : Relation entre Dmoe-B et Dmoe-G'; b : Relation entre Dmoe-C'et Dmoe-G'.

$\overrightarrow{\mathrm{MG}}$. est de $1,5 \mathrm{~cm}$ avec un écart type de $1,4 \mathrm{~cm}$. La forme plutôt régulière des rondelles et le grand nombre de rayons utilisés pour la décrire contribuent à réduire l'écart entre les deux centres de gravité.

\subsection{Calcul de l'excentricité}

L'excentricité désigne l'écart entre la moelle et le centre de gravité réel de la sur- face de la rondelle. Utiliser le centre de gravité (B) des points ( $\mathrm{Ai}$ ) conduit automatiquement à une erreur puisqu'il dépend fortement de la position du centre des rayons équirépartis en direction. Si ce dernier est situé sur la moelle, l'excentricité est sousestimée (environ deux fois moins, figure $5 a$ ).

La figture 6 montre comment évoluent, en fonction du nombre de rayons utilisés, les estimations de la surface de la rondelle et la position des estimateurs G'et C'. Les 


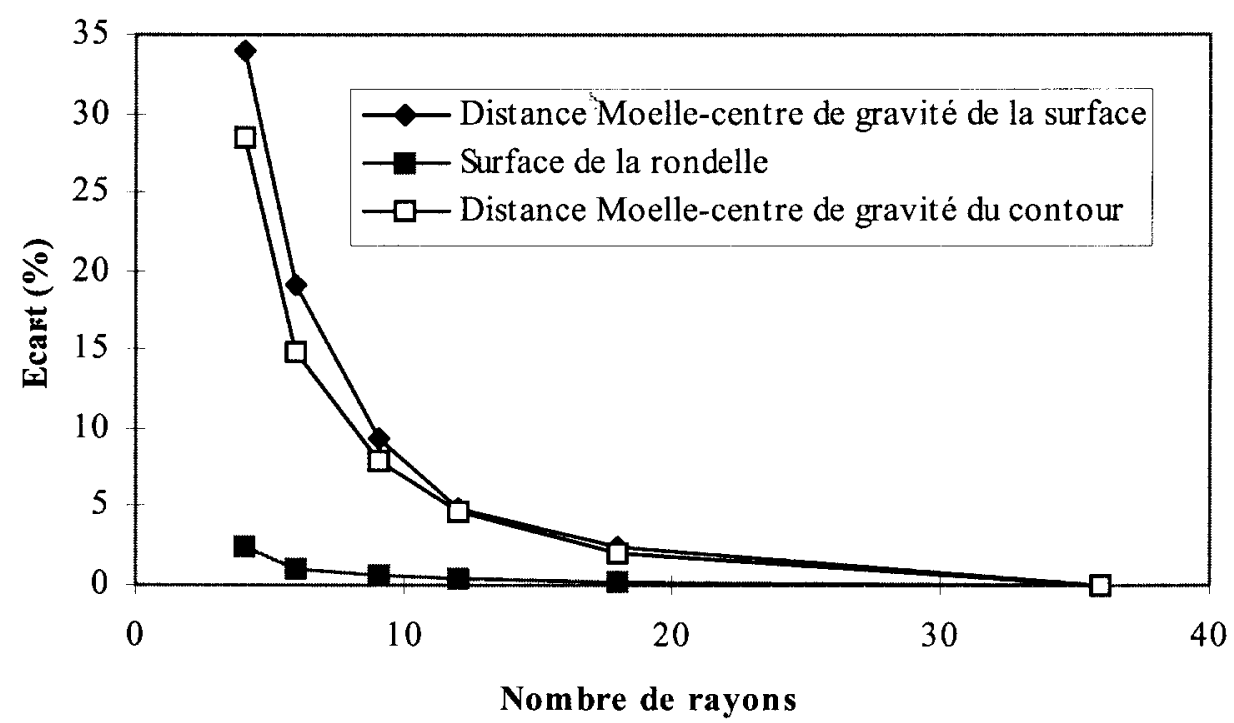

Figure 6. Erreur relative sur la surface d'une rondelle et sur la distance de la moelle aux centres de gravité de la surface et du contour, estimés à l'aide d'un certain nombre de rayons équirépartis en angle et originaires de la moelle.

écarts sont calculés par rapport aux valeurs de référence obtenues avec 36 rayons que l'on suppose peu différentes des valeurs réelles. Il apparaît que quatre rayons orthogonaux $(n=2)$ et originaires de la moelle suffisent amplement pour estimer la surface d'une rondelle

$\left(\operatorname{avec} S=\frac{\pi}{2 n} \sum_{i=1}^{2 n} M A_{i}^{2}\right)$.

$75 \%$ des estimations présentent un écart inférieur à $3 \%$ de la valeur de référence (94\% lorsque six rayons sont utilisés). Ce résultat est conforme à ce que l'on peut trouver dans la littérature à ce sujet. Pardé \& Bouchon [16] montrent par exemple que dans le cas des projections de houppiers avec huit rayons, $95 \%$ des estimations ont un écart inférieur à $3 \%$ de la surface mesurée par planimétrie. Cette information est importante dans les cas des analyses de tiges où l'objectif est notamment d'estimer la surface des cernes.

En revanche, lorsqu'il s'agit d'estimer l'excentricité, il est nécessaire d'utiliser au moins 18 rayons pour avoir la même précision de $3 \%$ sur l'estimation de la distance Moelle - Centre de gravité de la surface. Ce résultat n'est pas étonnant dans la mesure où il faut décrire assez précisément la forme de la rondelle pour positionner correctement ce point. Néanmoins, avec seulement quatre rayons orthogonaux, la moyenne des écarts est de $35 \%$, ce qui reste nettement inférieur à la valeur de l'ordre de $50 \%$ obtenue lorsqu'on utilise le centre de gravité des 36 points B.

\subsection{Calcul du méplat}

Un critère simple de forme est le méplat c'est-à-dire le rapport entre le plus grand diamètre passant par le «centre de gravité » 
et son perpendiculaire. En reprenant le cas de l'ellipse, la figure 7 montre la localisation de ces diamètres (D1', D2') en utilisant le centre de gravité des points $\mathrm{B}$ ou le centre de gravité de la surface G'(D1, D2) tous deux calculés avec 36 rayons.

Le méplat réel de l'ellipse est de 1,865 (grand axe/petit axe). En utilisant G', la valeur obtenue est très proche, D1/D2 $=1.869$, tandis que l'utilisation du centre de gravité des points surestime l'aplatissement de l'ellipse (D1'/D2'=1,938).

La figure 8 compare les valeurs de méplat obtenues avec le centre de gravité des points (B) et le centre de gravité de la surface (G') pour les 168 rondelles et en utilisant 36 rayons. Il n'existe pas de différence systématique, même pour les rondelles fortement aplaties. L'écart maximal entre les deux valeurs de méplat est de $8 \%$.

\section{CONCLUSION}

Cette note a permis d'expliciter les problèmes posés par l'estimation du centre de gravité d'une section à partir de rayons équirépartis en angle et originaires de la moelle. Le centre de gravité des points habituellement utilisé dans la littérature, génère des erreurs sur le calcul de l'excentricité (50\%) et dans une moindre mesure sur l'estimation du méplat ( $8 \%$ au maximum). Il est donc préférable d'utiliser le centre de gravité de la surface ou du contour, c'est à dire pondérer dans un cas par la surface et dans l'autre cas par la longueur des segments.

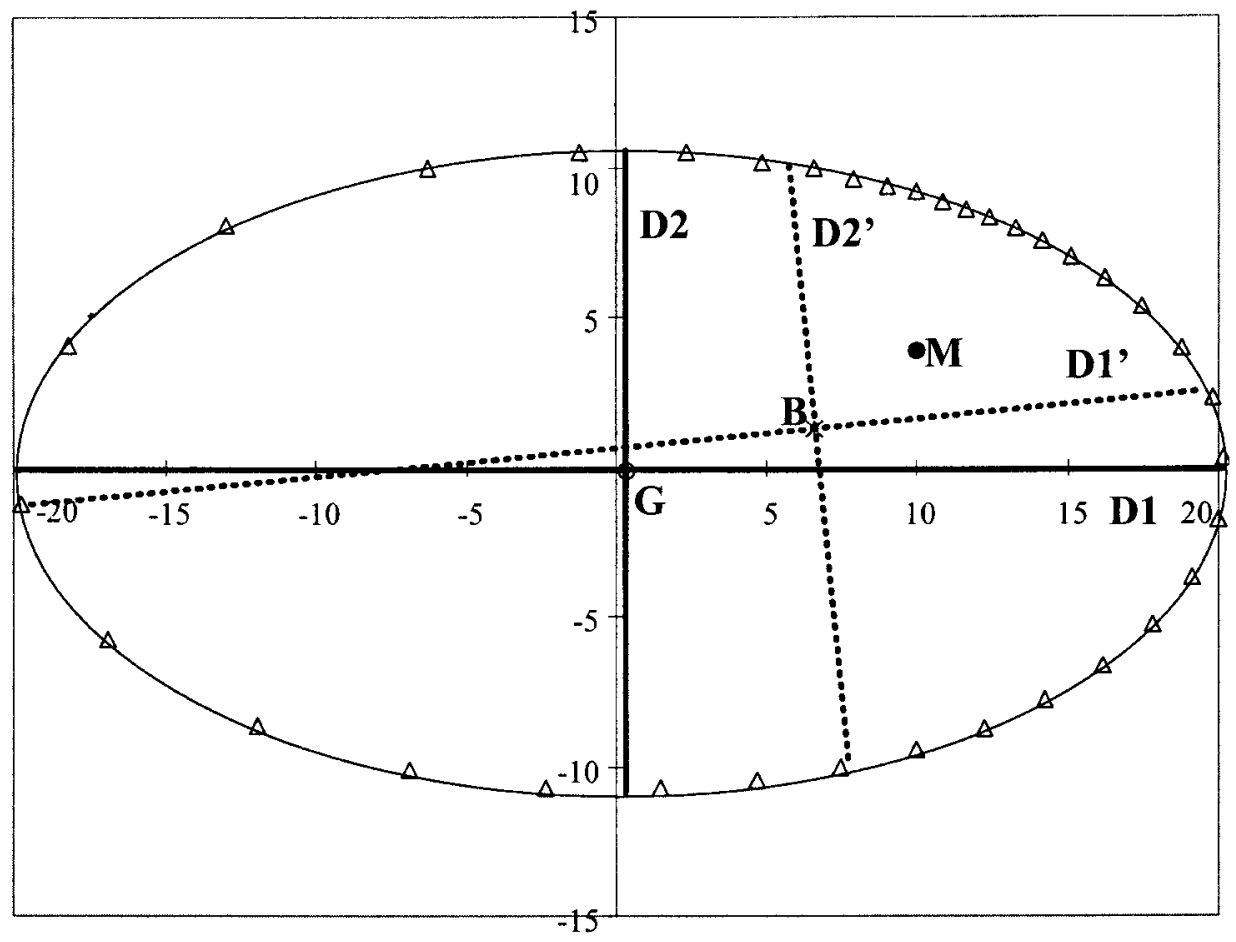

Figure 7. Diamètres utilisés pour le calcul du méplat avec le centre de gravité des points (B) et avec le centre géométrique (G'), dans le cas d'une ellipse. 


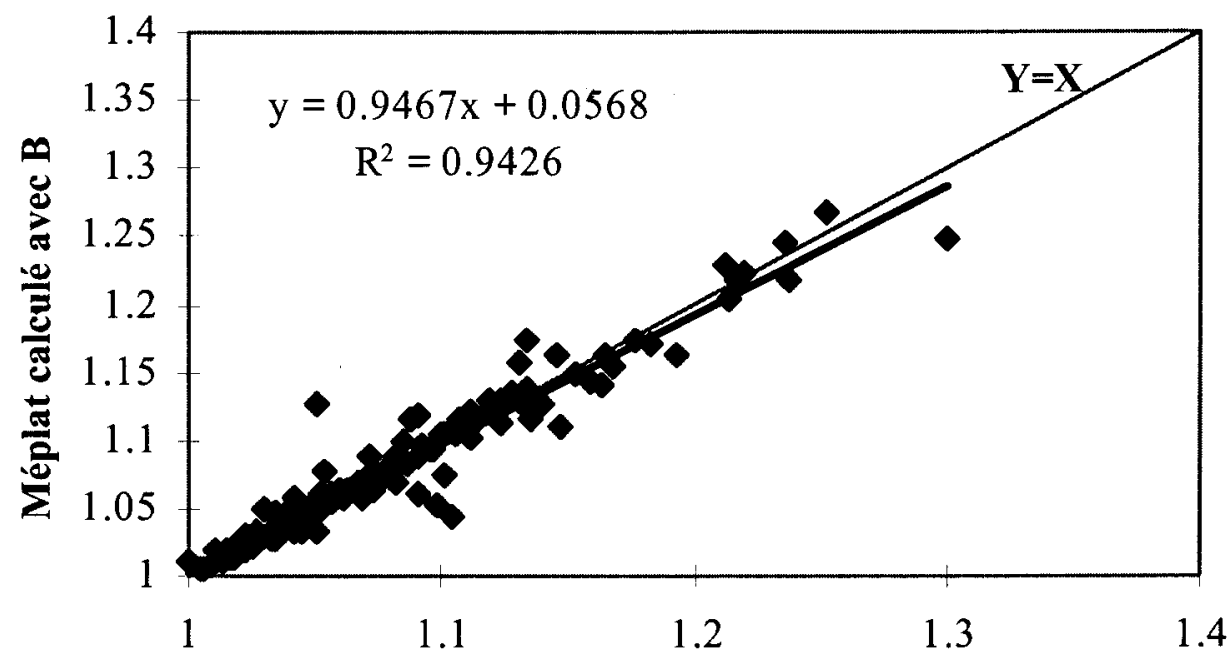

Méplat calculé avec G'

Figure 8. Comparaison entre le méplat calculé avec le centre de gravité des points (B) et celui calculé avec le centre géométrique $\left(G^{\prime}\right)$, sur 168 rondelles.

L'analyse qui est faite dans cet article montre que C'et G'sont pratiquement confondus lorsque le nombre de rayons est élevé, en revanche, il n'y a pas d'éléments qui permettent de choisir entre C'et G'lorsque le nombre de rayons est faible. En moyenne, C'converge plus vite vers le centre de gravité réel de la rondelle mais cet estimateur est sensible à l'orientation des rayons lorsque la rondelle est méplate et lorsque son contour est perturbé.

Dans le cas où les rayons ne sont pas équirépartis en angle mais choisis de telle façon que la distance entre deux points sur le contour soit constante (en curviligne), le centre de gravité des points tend bien vers le centre de gravité du contour lorsque le nombre de points tend vers l'infini. Cependant, cette méthode est difficile à mettre en œuvre notamment pour des rondelles perturbées (empattement, fourche), et les calculs de surface ne sont plus aussi simples puisque l'angle entre deux rayons n'est pas constant.
La question du choix de la moelle comme point d'origine des rayons peut se poser dans le cas de rondelles au contour irrégulier ou très excentrées. Si l'objectif est d'estimer avec une grande précision la surface ou la position du centre de gravité de la rondelle alors il est préférable de positionner l'origine des rayons le plus près possible du centre de la rondelle et non sur la moelle. En revanche, lorsque l'objectif est d'étudier la dynamique de la croissance en grosseur des arbres, la moelle est le seul point fixe de la rondelle et s'impose naturellement comme origine des rayons.

Dans le cas des analyses de tige classiques, positionner le centre de gravité de la surface ou du contour d'une rondelle nécessite un nombre important de mesures de rayons. Une autre possibilité, fiable et facile à mettre en œuvre est de calculer ces centres de gravité par analyse d'image. La surface scannée de la rondelle est entièrement décrite par un ensemble de pixels et les problèmes dus à la discrétisation du 
contour ne se posent plus. Par contre, la difficulté est de détecter les limites de cernes et la position de la moelle.

Pour terminer, une meilleure estimation de l'excentricité peut contribuer à améliorer les algorithmes d'optimisation de débit utilisés en scierie. En effet l'asymétrie de la section interne de grumes se traduit par des variations de largeur des cernes, élément important pour les propriétés du bois. Enfin, l'étude des variations de forme de sections transversales de tronc au cours de la croissance passée des arbres donnera des informations sur les variations de l'excentricité au cours du temps ce qui pourra conduire à la prendre en compte éventuellement dans les modèles de croissance d'arbres.

\section{REMERCIEMENTS}

Cette étude est réalisée dans le cadre du projet Européen « Improved Spruce Timber Utilisation » FAIR CT 96-1915 coordonné par le professeur $\mathrm{G}$. Lönner. Je tiens également à remercier les deux lecteurs des Annales des Sciences Forestières pour leur lecture attentive et pertinente.

\section{RÉFÉRENCES}

[1] Barger R.L., Folliot P.F., Factors affecting occurence of compression wood in individual Ponderosa Pine trees, Wood Sci. 8 (1976) 201-208.

[2] Biging G.S., Wensel L.C., The effect of eccentricity on the estimation of basal area and basal area increment of coniferous trees, For. Sci. 34 (1988) 621-633.

[3] Bindzi I., Samson M., Kamoso L.M., Modélisation géométrique d'une bille de bois, Ann. Sci. For. 53 (1996) 21-30.

[4] Boissieras A., Recherche de prédicteurs juvéniles de l'aptitude génétique à la croissance en volume chez le pin maritime. Détermination de l'âge optimal pour la sélection. Mémoire de stage option « forêt 》, école forestière de Meymac, Inra, département Forêt Bordeaux, 1984, 32p.

[5] Bouillet J.P., Méthode pour caractériser l'irrégularité de la forme des tiges en section transversale et son évolution au cours du temps, Ann. Sci. For. 50 (1993) 187-203.

[6] Bouillet J.P., Houllier F., Influence des éclaircies sur la forme de la section droite du tronc de Pinus Kesiva dans la région de Mangoro (Madagascar), Ann. Sci. For. 51 (1994) 267-281.

17] Chanson B., Dynamique de l'élaboration du bois : Nature et disposition dans la structure arborée. $5^{\mathfrak{c}}$ Séminaire interne, Architecture, Structure, Mécanique de l'arbre, Paris, éditeur LMGC, université Montpellier II, 1992, pp. 39-55.

[8] Daniels F.W., Schutz C.J., Regular planting patterns in Pinus Patula stands in the eastern transvaal, For. S. Af. 16 (1975) 61-62.

[9] Fonweban J.N., Houllier F., Tarifs de cubage et fonctions de défilement pour Eucalyptus saligna au Cameroun, Ann. Sci. For. 54 (1997) 513-528.

[10] Koch P., Coté W.A. Jr, Schlieter J., Day A.C., Incidence of compression wood and stem eccentricity in Lodgepole Pine of North America, USDA For. Serv. Intermountain Research Station, Research paper INT. 420 (1990) $42 \mathrm{pp}$.

[11] Laar A.V., The shape of the cross-section of tree boles in the western cape province, For. S. Af. 2 (1963) $71-78$.

[12] Leban J.M., Duchanois G., SimQUA : un logiciel de simulation de la qualité des bois, Ann. Sci. For. 47 (1990) 483-493.

[13] Loup C., Fournier M., Chanson B., Relations entre architecture, mécanique et anatomie de l'arbre. Cas d'un Pin Maritime (Pinus Pinaster Soland.). In : L'arbre. Biologie et développement. Naturalia Monspeliensa, 1991, pp.181-195.

[14] Matern B., On the shape of the cross-section of a tree stem, Avdelningen för skoglig biometri, Swedish university of agricultural sciences, section of forest biometric, rapport $n^{\circ}$ UMEA 1990, 28 (1990) $\mathrm{I}-47$.

[15] Newnham R.M., Variable-form taper functions for four Alberta tree species, Can. J. For. Res. 22 (1992) 210-223.

[16] Pardé J., Bouchon J., Dendrométrie. $2^{c}$ édition, Engref Nancy, 1988.

[17] Pawsey C.K., Lean and eccentricity in Pinus radiata (D.Don) in the south east of South Australia, Aus. For. Res. 2 (1966) 22-35.

[18] Radi M., Analyse morphologique de l'arbre en vue de sa modélisation mécanique, thèse, Université Bordeaux I, N809, 1992, $155 \mathrm{pp}$.

[19] Schweingruber F.H., Tree rings. Basics and applications of dendrochronology, Kluwer Academic Publishers, 1989, 276 pp.

[20] Williamson R.L., Out-of-roundness in Douglas-fir stems, For. Sci., $21: 4$ (1975) 365-370. 